\title{
Monocyte Activation in Angiogenesis and Collateral Growth in the Rabbit Hindlimb
}

\author{
Margarete Arras, Wulf D. Ito, Dimitri Scholz, Bernd Winkler, Jutta Schaper, and Wolfgang Schaper \\ Department of Experimental Cardiology, Max-Planck Institute for Physiological and Clinical Research, D-61231 Bad Nauheim, Germany
}

\begin{abstract}
We have previously shown that monocytes adhere to the vascular wall during collateral vessel growth (arteriogenesis) and capillary sprouting (angiogenesis). In this study we investigated the association of monocyte accumulation with both the production of the cytokines-basic fibroblast growth factor (bFGF) and TNF- $\alpha$-and vessel proliferation in the rabbit after femoral artery occlusion. In particular, we studied the effects of an increase in monocyte recruitment by LPS on capillary density as well as collateral and peripheral conductance after $7 \mathrm{~d}$ of occlusion. Monocytes accumulated around day 3 in collateral arteries when maximal proliferation was observed, and stained strongly for bFGF and TNF- $\alpha$. In the lower limb where angiogenesis was shown to be predominant, macrophage accumulation was also closely associated with maximal proliferation (around day 7). LPS treatment significantly increased capillary density $\left(424 \pm 26.1 \mathrm{n} / \mathrm{mm}^{2}\right.$ vs. $312 \pm 20.7 \mathrm{n} / \mathrm{mm}^{2} ; P<$ $0.05)$ and peripheral conductance $(109 \pm 33.8 \mathrm{ml} / \mathrm{min} / 100$ $\mathrm{mmHg}$ vs. $45 \pm 6.8 \mathrm{ml} / \mathrm{min} / 100 \mathrm{mmHg} ; P<0.05)$ as compared with untreated animals after $7 \mathrm{~d}$ of occlusion. These results indicate that monocyte activation plays a major role in angiogenesis and collateral artery growth. (J. Clin. Invest. 1997. 40-50.) Key words: collateral arteries • fibroblast growth factor • tumor necrosis factor - lipopolysaccharide • macrophages
\end{abstract}

\section{Introduction}

In the adult organism, vessels grow either via capillary sprouting, a process called angiogenesis (1), or via in situ proliferation of preexisting arteriolar connections into true collateral arteries, a process we named arteriogenesis $(2,3)$. Although capillary sprouting may deliver some relief to the underperfused territory, only true collateral arteries are principally capable of providing large enough amounts of blood flow to the

Margarete Arras and Wulf D. Ito contributed equally to this study. This work was presented in part at the 69th scientific session of the American Heart Association (1996. Circulation. 94:I-608).

Address correspondence to Dr. Wulf Ito, M.D., Department of Experimental Cardiology, Max-Planck Institute for Physiological and Clinical Research, Benekestrasse 2, D-61231 Bad Nauheim, Germany. Phone: +49-6032-705-402; FAX: +49-6032-705-419; E-mail: wito@alpha.kerckhoff.mpg.de

Received for publication 4 February 1997 and accepted in revised form 17 October 1997.

J. Clin. Invest.

(C) The American Society for Clinical Investigation, Inc. 0021-9738/98/01/0040/11 \$2.00

Volume 101, Number 1, January 1998, 40-50

http://www.jci.org ischemic area at risk for necrosis or loss of function. Both processes occur in the heart upon progressive vessel occlusions. Depending on the model studied, either angiogenesis or arteriogenesis predominates $(2,4)$. We recently described a model in the rabbit hindlimb in which both processes could be studied simultaneously (3). In this model, collateral growth (arteriogenesis) was restricted to the thigh, whereas capillary sprouting (angiogenesis) was observed in the calf muscles upon femoral artery occlusion. Both arteriogenesis and angiogenesis were rapidly initiated and showed maximum proliferation during the first week after arterial occlusion, suggesting that growth factors were almost instantly provided in sufficient amounts. To explain the rapid onset and early intensity of vascular growth, we studied and modified the behavior of monocytes that adhere to the stressed vessel wall, transmigrate, and produce growth factors and cytokines that in an autocrine fashion, attract additional monocytes.

We recently demonstrated that local infusion of monocyte chemoattractant protein-1 (MCP-1) ${ }^{1}$ markedly increased collateral and peripheral conductance within $7 \mathrm{~d}$ after femoral artery occlusion. This finding supports the hypothesis that monocyte accumulation is a critical player in both capillary spouting (angiogenesis) and collateral artery growth (arteriogenesis; 5). Monocytes are known to produce a variety of cytokines upon activation (6-9). Production of basic fibroblast growth factor (bFGF) and TNF- $\alpha$, cytokines with known angiogenic properties, belongs to the repertoire of monocytes (10-12).

bFGF has been shown to potentiate the effect of vascular endothelial growth factor (VEGF), and in contrast to VEGF also acts directly on smooth muscle cells $(13,14)$. Indirect effects of VEGF on smooth muscle cells very likely involve bFGF. bFGF lacks a signal sequence and cannot be secreted using the classical pathways. Recently, however, it was shown that despite the lack of a signal sequence, bFGF is able to leave the producing cell $(15,16)$. Migrated monocytes also die in situ, thereby releasing bFGF.

TNF- $\alpha$ is a pleiotropic cytokine produced primarily by activated macrophages, and is a major mediator of inflammatory reactions. It has not only been shown to be angiogenic in vivo (10), but inhibition of TNF- $\alpha$ has also been demonstrated to reduce angiogenesis (17). In particular, $\mathrm{TNF}_{-\alpha}$ is responsible for adhesion and activation of additional monocytes via upregulation of cell adhesion molecules on both endothelial cells and monocytes, and by upregulation of $\operatorname{GM}-\operatorname{CSF}(18,19)$. The known angiogenic properties of this cytokine may therefore be explained in part by the augmentation of the monocytic response.

LPS is a strong inducer of TNF- $\alpha$ production as previously shown $(20,21)$. After a single injection of LPS in the rabbit,

1. Abbreviations used in this paper: $\mathrm{bFGF}$, basic fibroblast growth factor; BrdU, bromodeoxyuridine; MCP-1, monocyte chemoattractant protein 1; VEGF vascular endothelial growth factor. 
$\mathrm{TNF}-\alpha$ plasma levels rise sharply within $3 \mathrm{~h}$ and remain elevated for $24 \mathrm{~h}$. After a delay of 6-12 h, the number of TNF$\alpha$-containing macrophages increases more than fivefold in all organs investigated. Rarely were other cell types found to produce TNF- $\alpha$ in response to LPS injection (21).

We asked whether monocytes accumulate and produce bFGF and TNF- $\alpha$ at the time of maximal proliferation of collateral arteries and capillaries as an explanation for the rapid onset and early intensity of collateral vessel proliferation. Functional involvement of TNF- $\alpha$ in angiogenesis and collateral growth was suggested by studies in which the production of TNF- $\alpha$ was stimulated via LPS injection.

\section{Methods}

Animal model. This study was performed with permission of the State of Hesse, Regierungspräsidium Darmstadt, according to section 8 of the German Law for the Protection of Animals. It conforms with the Guide for the Care and Use of Laboratory Animals (National Institutes of Health publication no. 85-23, revised 1985). A total of 39 rabbits were investigated in this study. For histological investigations, 18 rabbits were divided into 6 groups of equal size. 12 rabbits were randomly assigned to either $7 \mathrm{~d}$ or no femoral artery occlusion either with or without LPS treatment. Six animals were supplied with an osmotic minipump (2ML-2; Alza Corp, Palo Alto, CA) delivering bromodeoxyuridine (BrdU, Sigma Chemical Co., St. Louis, MO) via a catheter in the carotid artery to study proliferation of collateral arteries and capillaries. They were subjected to either 3 or $7 \mathrm{~d}$ of femoral artery occlusion.

Five groups of animals were investigated in the hemodynamic study. 12 rabbits were subjected to $7 \mathrm{~d}$ of bilateral femoral artery occlusion. They were randomly assigned to receive either ( $a$ ) LPS intravenously $3 \mathrm{~d}$ after occlusion, or $(b)$ no treatment. Nine additional animals were subjected to no, acute, or $21 \mathrm{~d}$ of femoral artery occlusion for comparison. Hindlimbs were evaluated separately because the variation of conductances was not lower in hindlimbs of the same as compared with hindlimbs of different animals as described previously (3). Perfusion was only performed in hindlimbs in which adequate peripheral pressures were measurable in vivo.

For the initial surgery, the animals were anesthetized with an intramuscular injection of ketamine hydrochloride $(4-8 \mathrm{mg} / \mathrm{kg}$ body weight) and xylazine (8-9 $\mathrm{mg} / \mathrm{kg}$ body weight). Supplementary doses of anesthetic (10-20\% of the initial dose) were given intravenously as needed. Surgery was performed under sterile conditions. Femoral arteries were exposed and closed with two ligatures about $2 \mathrm{~cm}$ apart, leaving the branching of the arteria profunda femoris, the arteria circumflexa femoris lateralis, and the arteria circumflexa abdominis patent. Wounds were closed and draped. Rabbits were outfitted with a specially designed body suit that prevented self-mutilation, but did not restrict normal activities. They were housed together in a large cage to secure mobility, and had free access to water and chow.

LPS from Escherichia coli (serotype O113; Assoc. of Cape Cod, Inc., Woods Hole, MA) was dissolved to give a final concentration of $5 \mu \mathrm{g} / \mathrm{ml}$ in saline for intravenous injections. LPS was given as a bolus $(1 \mu \mathrm{g} / \mathrm{kg}$ body wt in $2 \mathrm{ml} \mathrm{NaCl} 0.9 \%$ ) into the ear vein at day 3 after femoral artery occlusion.

Before death the animals received another intramuscular injection of ketamine hydrochloride and xylazine. They then underwent tracheostomy, and were artificially ventilated. Anesthesia was deepened with pentobarbital $(12 \mathrm{mg} / \mathrm{kg}$ body $\mathrm{wt} / \mathrm{h})$. The carotid artery was cannulated for continuous pressure monitoring.

All animals survived the primary operation and recuperated quickly. We did not observe any gangrene or gross impairment of function after femoral artery occlusion. All animals assigned to histological investigations entered the study. Three animals of the group assigned to hemodyamic evaluations had to be excluded from the study because of air embolism. 2 of the total 25 perfused hindlimbs were excluded because peripheral pressures could not be obtained.

Perfusion fixation and preparation of histological samples. In animals assigned to histological examination, the abdominal aortas were cannulated with a 2-mm bore metal canula, the chest was opened, and the heart was exposed. After incision of the right atrium for drainage, perfusion was started with a rinsing solution containing $0.5 \% \mathrm{BSA}$, $5 \mathrm{mM}$ EDTA, and $0.317 \mathrm{mg} /$ liter adenosine in PBS for $5 \mathrm{~min}$ followed by fixation with formalin ( $4 \%$ in the rinsing solution without BSA) for $20 \mathrm{~min}$. Subsequently, a postmortem angiography was performed (as described below), which allowed precise localization and excision of collateral vessels, their stem, and reentry regions. Neighboring vessels of the same size served as controls. Samples from the gastrocnemial and peroneal muscles were also obtained. Samples were kept for cryoprotection in $20 \%$ saccharose in PBS overnight, and then frozen in methyl butane and stored at $-80^{\circ} \mathrm{C}$.

Hemodynamic evaluation. In rabbits assigned to hemodynamic measurements, the arteria saphena magna (anterior tibial artery in humans; main arterial supply to the lower limb and foot in the rabbit) was exposed just above the ankle and cannulated with polyethylene tubing ( $0.58 \mathrm{~mm}$ i.d., $0.96 \mathrm{~mm}$ o.d.) connected to a P23DC pressure transducer (Statham Instruments, Hato Rey, PR) for measurement of peripheral pressures. After heparinization with 5,000 $\mathrm{U}$ of heparin, both external iliac arteries were exposed and cannulated with a 2.0$\mathrm{mm}$ bore metal tube. The abdominal circumflex artery and the arteria spermatica were ligated, and a tourniquet was placed proximally around both thighs leaving the femoral artery patent. The femoral and sciatic veins were incised for venous blood drainage. The animals then were bled, and the legs were amputated above the hip and quickly transferred to the perfusion apparatus.

Ex vivo pressure-flow relations. The legs were perfused with autologous oxygenated blood warmed to $37^{\circ} \mathrm{C}$ using a roller pump (Stoeckert GmbH, Munich, Germany) and an M2 membrane oxygenator (Jostra GmbH, Hirrlingen, Germany). Hematocrit was kept between 34 and 37\%, and oxygen saturation was kept at 99\%. Maximal vasodilation was achieved by adding $25 \mathrm{mg}$ of papaverine (Sigma Chemical Co., St. Louis, MO) to the perfusate (priming volume, 60 $\mathrm{ml})$. The legs were perfused at three different pressures $(40,60$, and $80 \mathrm{mmHg}$ ). After stabilization, radioactive microspheres were injected, and a reference sample was drawn using a syringe pump (Braun Melsungen, Melsungen, Germany). For each pressure level, microspheres labeled with Ruthenium, Cerium, Niobium, or Scandium (Dupont-NEN, Boston, MA) were randomly chosen to allow measurement of tissue perfusion at different perfusion pressures. Total flow was determined using an ultrasonic in-line flow probe connected to a T201 flowmeter (Transonic Systems, Inc., Ithaca, NY). Systemic pressures and peripheral capillary pressures were traced with a P23DC pressure transducer (Statham). All recordings were transferred on-line to a computerized recording system (MacLab; Apple AD Instruments, Castle Hill, Australia) from which they were recovered for further processing.

Microsphere Counting. Quadriceps, adductor longus and adductor magnus, gastrocemius, soleus, and peroneal muscles were dissected from the leg, and each muscle was divided into five consecutive samples from the proximal to the distal end. Samples were weighed and subsequently analyzed together with the respective reference samples using a Ge-detector as described previously (22). Calculation of flows and conductances was performed as described previously (3).

Post mortem angiography. After drug-induced vasodilation, legs were perfused with warm $\left(37^{\circ} \mathrm{C}\right) \mathrm{Krebs}$-Henseleit buffer for $1 \mathrm{~min}$ followed by perfusion with warm contrast medium based on bismuth and gelatin (23). Subsequently, the contrast medium was allowed to gel by placing the limb on crushed ice, and angiograms were taken at two different angles in a radiography apparatus (Balteau, Belgium; tube from Machlett Laboratories) using a single enveloped Structurix D7 DW film (Agfa-Gevaert N.V., Belgium). The resulting stereoscopic images allowed three-dimensional analysis of collateral growth. 


\section{Immunohistochemistry}

Antibodies. To study proliferation, two different monoclonal antiBrdU antibodies were used (BU 20a; DAKO A/S, Glostrup, Denmark; 85-2C8; Neomarkers, Fremont, CA). Proliferation was also studied using a monoclonal antibody against KI 67 (MIP-5; Dianova, Hamburg, Germany). The monoclonal antibody RAM 11 (DAKO A/S) was used to visualize rabbit macrophages. In preliminary studies, we were able to show that this antibody that was raised against alveolar macrophages stains monocytes on blood smears as well as on tissue macrophages in liver and spleen. RAM 11 has been used to investigate monocyte/macrophage accumulation in experimental rabbit arteriosclerosis $(11,24)$. Capillaries were stained with a monoclonal antibody against CD 31/PECAM (JC/70A, DAKO A/S Denmark). For the study of bFGF, two different antibodies were used (FB-8; Sigma Chemical Co., FGF3; Biotrend, Cologne, Germany). TNF- $\alpha$ was detected by a monoclonal antibody (RD 979; Miles Inc., Kankakee, IL). All antibodies were mouse anti-human except for RAM 11, which is a mouse antibody specific for rabbit macrophages. For the doublestaining procedures, the monoclonal antibodies RAM 11 and 85-2C8 were directly coupled with the fluorescent stain Cy5 using the Fluorolink $^{\mathrm{TM}}$ monoclonal antibody labeling kit (Biological Detection Systems, Pittsburgh, PA).

Staining for proliferation, capillaries, and macrophages. Cryostat sections of $20 \mu \mathrm{m}$ were obtained with a CM 3000 cryotome (Leica Inc., Deerfield, IL), mounted onto silicone-coated slides, and incubated in $2 \mathrm{~mol} / \mathrm{liter} \mathrm{HCl}$ at $38^{\circ} \mathrm{C}$ for $20 \mathrm{~min}$. After rinsing in PBS three times for $5 \mathrm{~min}$, sections were incubated overnight at $4^{\circ} \mathrm{C}$ with primary antibodies (see above) in a moist chamber, and then for $4 \mathrm{~h}$ at room temperature with FITC-coupled donkey anti-mouse antibody (Dianova) as a detection system. For background staining, the myocytes were stained with a TRITC-coupled phalloidin antibody, which is a specific marker for actin. The preparations were washed $4 \times$ for 3 min in PBS between each step. After nuclear staining with 7-aminoactinomycin D (Molecular Probes, Inc., Eugene, OR), sections were mounted with Mowiol (Hoechst, Frankfurt, Germany). Tissues where the primary antibody was omitted served as a negative control.

Double staining for macrophages and growth factors. Cryostat sections of $20-\mu \mathrm{m}$ thickness were incubated overnight at $4^{\circ} \mathrm{C}$ with either antibody against bFGF or one specific antibody against TNF- $\alpha$ as a primary antibody, and then for $4 \mathrm{~h}$ at room temperature with FITC-coupled donkey anti-mouse antibody (Dianova) as a detection system. This incubation was followed by another overnight incubation at $4^{\circ} \mathrm{C}$ with a directly Cy5-coupled antibody for rabbit macrophages.

Double staining for proliferation and capillaries in the lower leg. Cryostat sections of $20-\mu \mathrm{m}$ thickness were incubated overnight at $4^{\circ} \mathrm{C}$ with an antibody against CD31 (PECAM, clone JC/70A), and then for $4 \mathrm{~h}$ at room temperature with FITC-coupled donkey anti-mouse antibody (Dianova). This incubation was followed by another overnight incubation at $4^{\circ} \mathrm{C}$ with a directly Cy5-coupled antibody for BrdU.

Fluorescence microscopy. Slides were viewed in a DM microscope (Leica) and photographed with a TCS laserconfocal microscope with corresponding filters (Leica). Micrographs were taken with 100 ASA color slide film (Eastman Kodak Co., Rochester, NY). All pictures shown here are reproductions from slides.

Counting of capillaries and macrophages in the lower leg. Three samples were obtained from each peroneal and gastrocnemial muscle. Capillaries and macrophages were counted in up to 15 different sections, rendering mean capillary density and mean macrophage population per muscle. Counting was performed by a blinded observer, and was performed only on sections where capillaries were cut perpendicularly, and in tissue that was well-perfusion-fixed (i.e., capillaries seen as circles). Samples of one peroneal muscle had to be discarded because of poor fixation.

Statistical analysis. All data are presented as mean \pm SEM. Intergroup comparisons were performed by unpaired Student's $t$ test. The Mann-Whitney Rank Sum test was used in the case of unequal vari- ances. Probability values of 0.05 or less were required for assumption of statistical significance.

\section{Results}

Monocytes accumulate at about day 3 in collateral arteries in the thigh, and at about day 7 around proliferating capillaries in the calf. After femoral artery occlusion, collateral arteries develop between branches of the arteria circumflexa femoris lateralis and the arteriae genualis, as well as between branches of the arteria femoralis profunda and the arteria saphena parva. We observed that these collaterals were rapidly expanding by proliferation of their smooth muscle cells and endothelial cells during the first $3 \mathrm{~d}$ after femoral occlusion. This observation was confirmed $(a)$ with two different antibodies against BrdU that was infused during the first $3 \mathrm{~d}$ after femoral artery occlusion, and (b) by immunohistochemical staining for KI 67, an interphase protein that revealed marked proliferation of endothelial and smooth muscle cells at day 3 (Fig. 1, $b$ and $c$ ). Since BrdU shows accumulation of all cell divisions during BrdU infusion, the larger number of BrdU-positive cells compared with KI67-positive cells showed that significant proliferation had already occurred within the first $2 \mathrm{~d}$ after occlusion. At the same time, monocytes were adhering to and migrating through these collateral arteries, a process that was not seen at later time points (Fig. 1 a). Accumulation of monocytes was restricted to vessels identified as collateral arteries on stereoscopical angiograms, and by identification of the stem and reentry region in situ. Vessels of the same size taken from an adjacent normal region showed no accumulation of monocytes/ macrophages at any time point (Fig. $1 d$ ). Furthermore, the site of surgery was situated several centimeters away from the collateral arteries investigated, and the procedure itself only caused minimal tissue injuries. These facts exclude the possibility that the monocyte/macrophage accumulation seen in collateral arteries was due to nonspecific inflammatory reactions in this region.

Monocyte accumulation was also observed during angiogenesis. In contrast to collateral growth in the thigh, however, tissue macrophages were not seen to accumulate until $7 \mathrm{~d}$ after occlusion in the calf muscles where we observed angiogenesis (Fig. 2, $a$ and $b$ ). This observation correlated well with the late appearance of capillary proliferation in the lower limb (Fig. 2, $c$ and $d)$.

Monocytes are the main source of TNF- $\alpha$ and bFGF during collateral artery proliferation. Double staining of excised collateral arteries with antibodies specific for macrophages and bFGF revealed that this growth factor was almost exclusively found in monocytes at day 3 after femoral artery occlusion (Fig. 3, a-d). Identical staining patterns were observed with different antibodies against bFGF. Similar to bFGF, TNF- $\alpha$ was only observed in monocytes accumulating in collateral arteries $3 \mathrm{~d}$ after occlusion, but not in any other cells such as smooth muscle cells, endothelial cells, fibroblasts, or surrounding muscle cells (Fig. 4, $a-d$ ).

In contrast to the thigh, positive staining for bFGF in the lower limb was not confined to macrophages, but was also seen in many other cells after femoral artery occlusion (Fig. 5, $a-d$ ). Positive staining for TNF- $\alpha$, however, was rarely observed in this region, and was restricted to macrophages (results not shown).

The increase in capillary density is related to monocyte ac- 

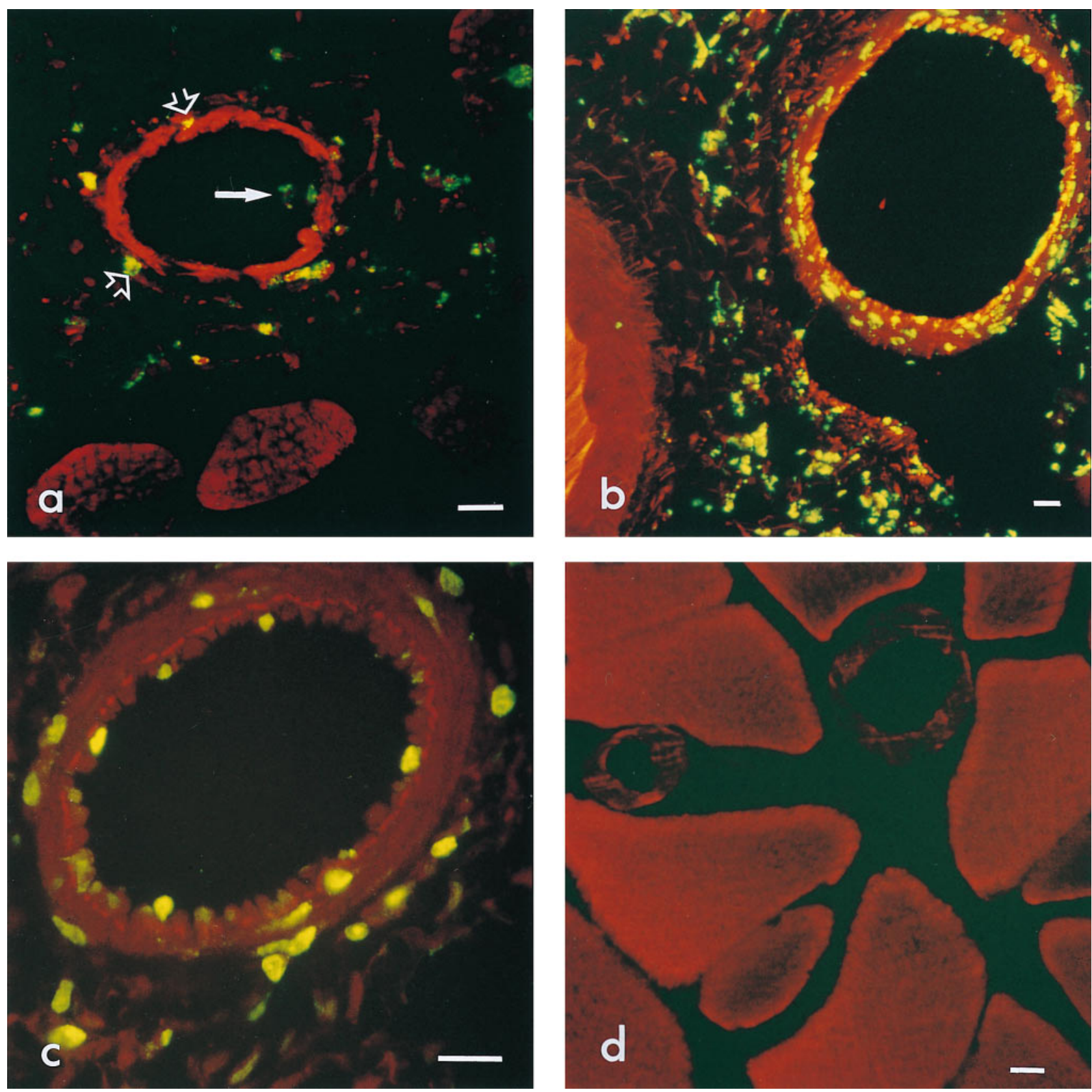

Figure 1. Monocyte accumulation and proliferation in excised collateral arteries after $3 \mathrm{~d}$ of occlusion. (a) Several macrophages are distributed in the adventitia and the connective tissue surrounding the vessels. Notice macrophages adhering to the endothelium (full arrow) and penetrating the wall (open arrows). (b) BrdU staining reveals massive proliferation of endothelial and smooth muscle cells. (c) KI 67 reveals proliferation of endothelial and smooth muscle cells at day 3. $(d)$ Similarly sized vessel adjacent to the excised collateral artery stained with an antibody against rabbit monocytes/macrophages. The absence of macrophage accumulation in neighboring control vessels indicates that the migration of monocytes is specific for collateral vessels. Bars, $20 \mu \mathrm{m}$.

cumulation. Counting capillaries in the lower limb revealed that proliferation of capillary endothelial cells, as visualized by double staining for CD 31 and BrdU, had produced a significant increase in the number of capillaries in the gastrocnemial muscle after $7 \mathrm{~d}$ of occlusion $\left(312 \pm 20.7 \mathrm{n} / \mathrm{mm}^{2}\right.$ vs. $221 \pm 10.3$ $n / \mathrm{mm}^{2}, P<0.05$; Fig. $2, c$ and $d$, and Fig. $6 a$ ). The increase in the number of capillaries in the gastrocnemial muscle corresponded to the increase in the number of monocytes during the first $7 \mathrm{~d}$ of femoral artery occlusion $\left(74 \pm 21.0 \mathrm{n} / \mathrm{mm}^{2} \mathrm{vs}\right.$. $11 \pm 5.5 \mathrm{n} / \mathrm{mm}^{2} ; P<0.01$; Fig. 6 b).

This result contrasted with the findings in the peroneal muscle, which was more densely vascularized under control conditions but failed to show an increase in the number of capillaries after occlusion $\left(369 \pm 9.9 n / \mathrm{mm}^{2}\right.$ vs. $367 \pm 95.3 \mathrm{n} / \mathrm{mm}^{2}$, NS; Fig. 7 a). Resident tissue macrophages were more numerous in the peroneal than in the gastrocnemial muscle in control 

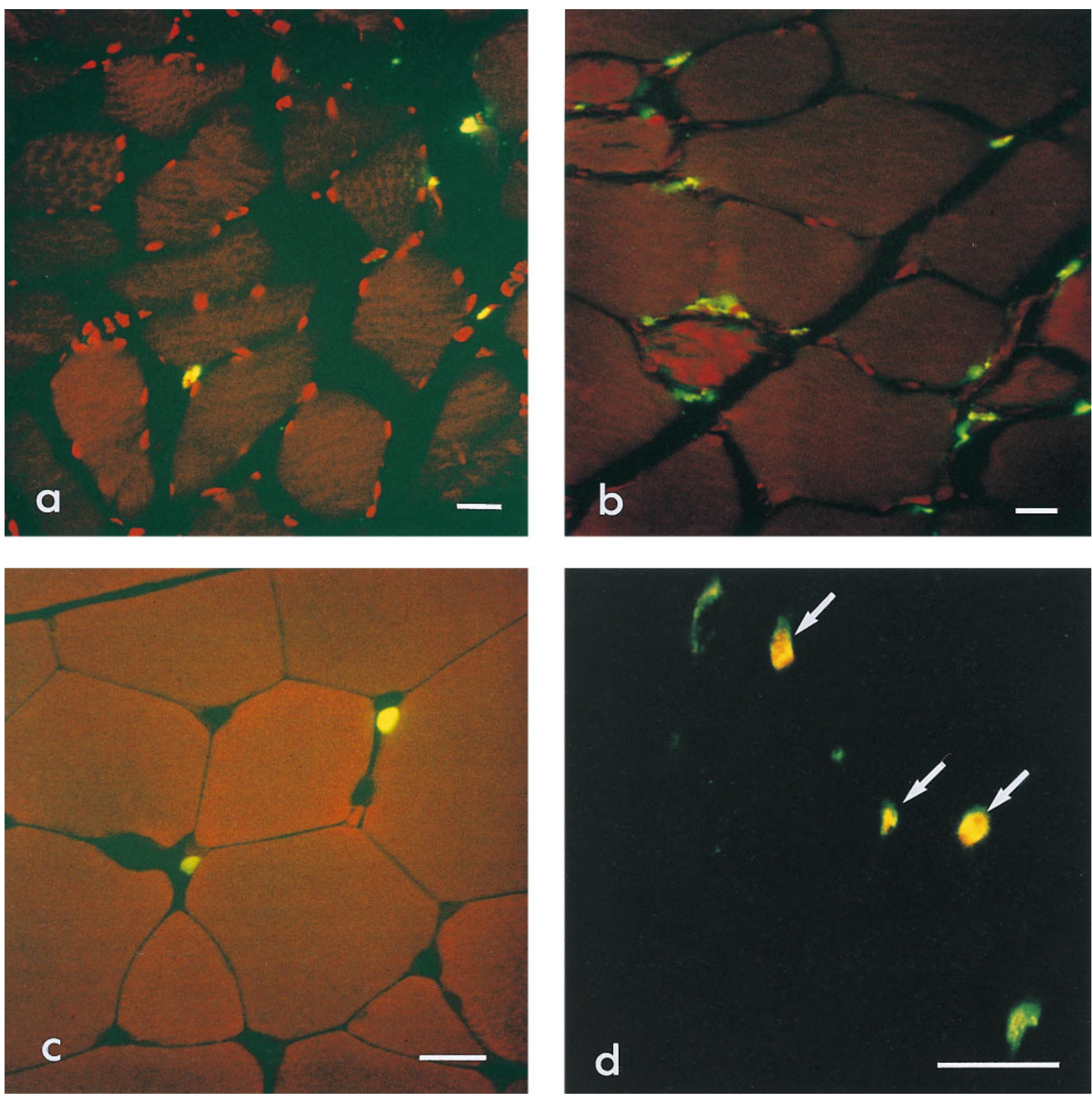

Figure 2. Monocyte accumulation and proliferation of capillaries in the calf muscles $7 \mathrm{~d}$ after occlusion. (a) Several macrophages are present in the interstitium of the gastrocnemial muscle. (b) Monocytes/ macrophages are more numerous after LPS treatment. (c) KI 67 staining demonstrates proliferation of cells in the interstitial space of the gastrocnemius muscle. (d) Double staining for BrdU and endothelial cells. Staining with and antibody for PECAM/CD31 in green shows capillaries, whereas red indicates BrdU in the nucleus of proliferating cells. Positive labeling for both antibodies in three endothelial cells (arrows) shows proliferation of capillaries in the lower leg $7 \mathrm{~d}$ after ligation of the femoral artery. Bars, $20 \mu \mathrm{m}$.

animals. Their numbers, however, did not increase upon femoral artery occlusion, correlating with the absence of angiogenesis in this muscle (Fig. $7 b$ ).

An increase of monocyte/macrophage accumulation by a single intravenous bolus of LPS leads to a significant increase in the number of capillaries in the calf muscles. Since accumulation and activation of monocytes occurred in relationship to angiogenesis and arteriogenesis, we examined whether augmentation of this monocytic response enhances collateral for- mation and angiogenesis. Intravenous injection of LPS at day 3 increased monocyte accumulation in both the gastrocnemial and the peroneal muscle after $7 \mathrm{~d}$ of occlusion (Figs. $6 b, 7 b$, and $2 b$ ). In both calf muscles, capillary density was higher in animals treated with LPS when compared with animals without treatment after $7 \mathrm{~d}$ of occlusion $\left(424 \pm 26.1 \mathrm{n} / \mathrm{mm}^{2}\right.$ vs. $312 \pm 20.7 \mathrm{n} / \mathrm{mm}^{2}, P<0.05$; and $1065 \pm 289.6 \mathrm{n} / \mathrm{mm}^{2}$ vs. $369 \pm 9.9$ $n / \mathrm{mm}^{2}$, NS; Figs. $6 a$ and $7 a$ ). LPS treatment also increased macrophage numbers and capillary density in hindlimbs not 

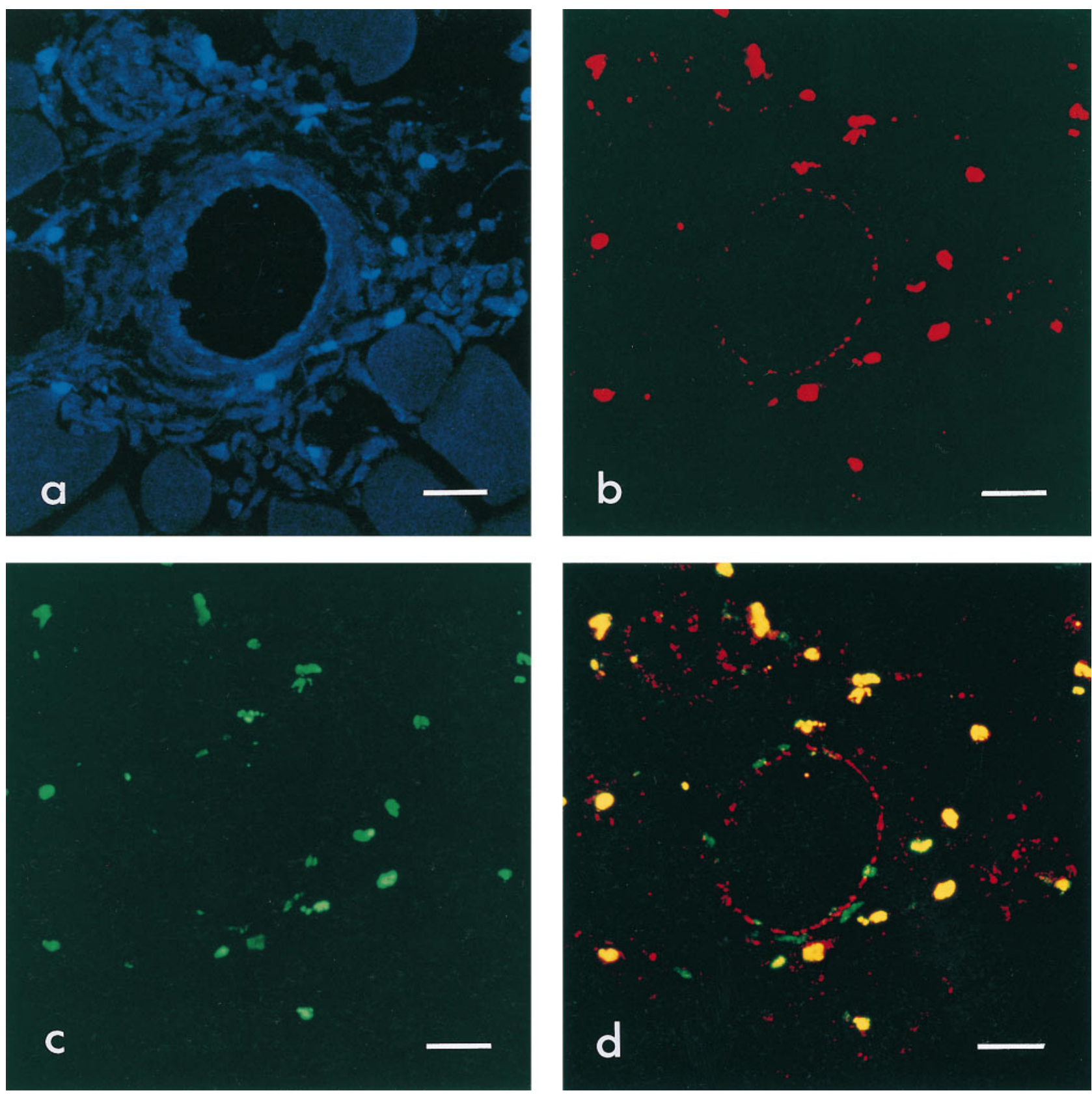

Figure 3. Double staining for bFGF and macrophages in the thigh. (a) Morphology of a collateral vessel in the thigh $3 \mathrm{~d}$ after ligation of the femoral artery. (b) The red channel shows specific staining for macrophages, and reveals labeling of several cells in all layers of the artery. (c) Using the green channel, specific staining for bFGF could be detected, and shows positive labeling of the same cells. $(d)$ Double staining for bFGF and macrophages in the same section shows several positive cells for both antibodies. The yellow staining is due to the overlapping of the red and green colors, showing macrophages as a source for bFGF. Bars, $20 \mu \mathrm{m}$.

subjected to femoral occlusion. The increase was $50 \%$ lower when compared with occluded hindlimbs, and was more pronounced in the peroneal muscle, indicating that the number of resident macrophages is crucial to the angiogenic effect of LPS (Fig. 6, $a$ and $b$, and Fig. 7, $a$ and $b$ ). These findings indicate that enhancement of monocyte accumulation by LPS injection is linked to an increase of angiogenesis.

Peripheral and collateral conductances are increased in LPS-treated animals as compared with control animals after $7 d$ of femoral artery occlusion. Functional evidence for an in- crease of capillary sprouting after injection of LPS was obtained from our hemodynamic studies. Hemodynamic evaluations were performed ex vivo for better control of the experiment. We observed a significant increase in peripheral conductance in animals receiving LPS when compared with control animals after $7 \mathrm{~d}$ of occlusion $(109 \pm 33.8 \mathrm{ml} / \mathrm{min} / 100$ $\mathrm{mmHg}$ vs. $45 \pm 6.8 \mathrm{ml} / \mathrm{min} / 100 \mathrm{mmHg}, P<0.05$; Fig. $8 a$ ). Since only capillaries were shown to proliferate in the calf, this increase in peripheral conductance was certainly due to capillary sprouting as described elsewhere (3). Although collateral con- 

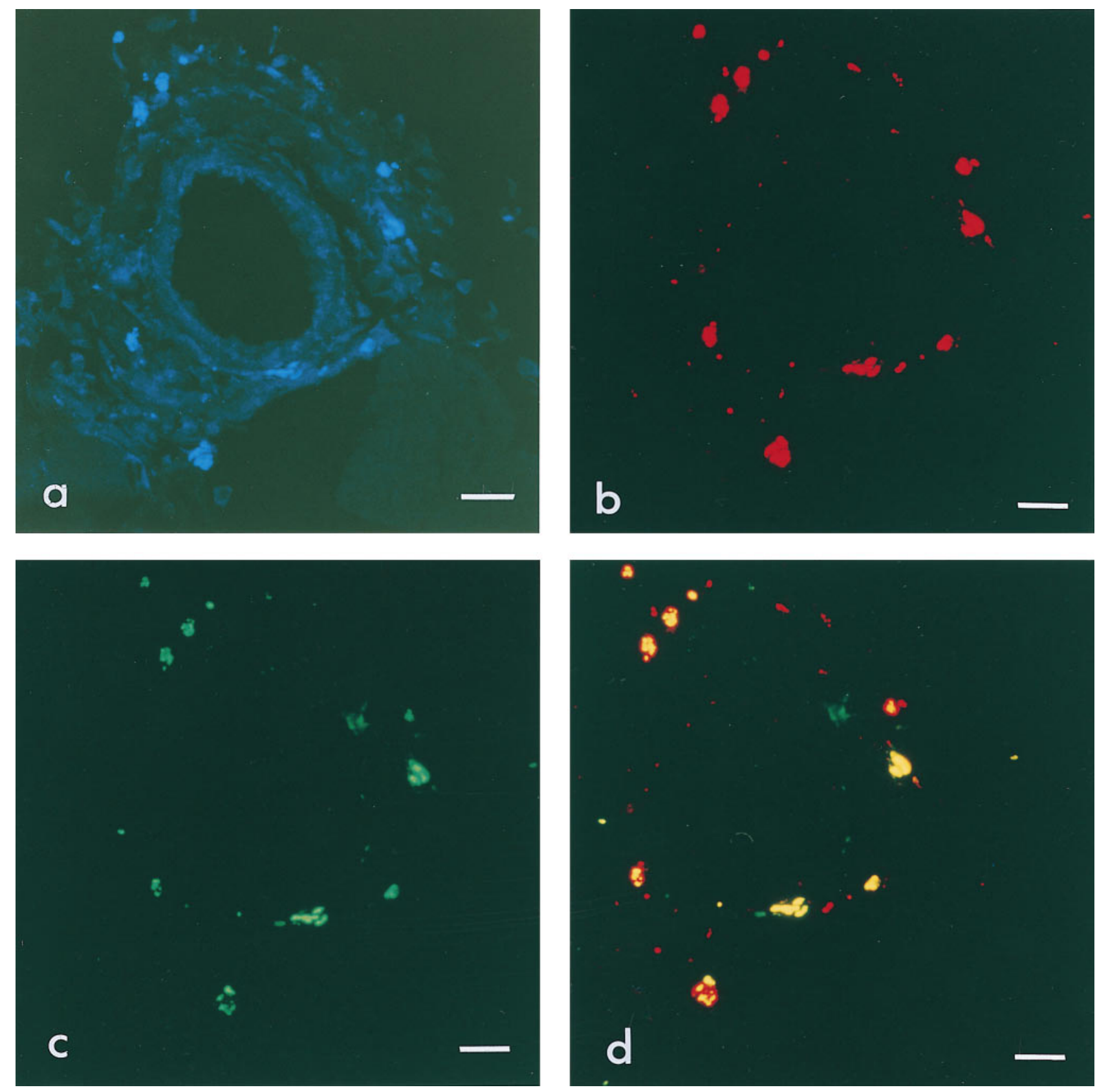

Figure 4. Double staining for TNF- $\alpha$ and macrophages in the thigh. (a) Morphology of a collateral vessel in the thigh $3 \mathrm{~d}$ after ligation of the femoral artery. (b) Several macrophages are visible after specific staining with RAM-11 in the adventitia of the vessel. (c) Specific staining for TNF- $\alpha$ in the same section shows an identical staining pattern. $(d)$ Double staining for TNF- $\alpha$ and macrophages in the same section shows several positive cells for both antibodies. The yellow staining is due to the overlapping of the red and the green colors, showing macrophages as main source for TNF- $\alpha$ in collateral vessels. Bars, $20 \mu \mathrm{m}$.

ductance also increased after LPS treatment, it did not reach statistical significance $(37 \pm 11.4 \mathrm{ml} / \mathrm{min} / 100 \mathrm{mmHg}$ vs. $25 \pm 2.5$ $\mathrm{ml} / \mathrm{min} / 100 \mathrm{mmHg}$, NS; Fig. $8 \mathrm{~b}$ ). Total conductance only showed a marginal increase (Fig. $8 c$ ).

\section{Discussion}

In this study we provide histological and functional data to show that monocyte accumulation and activation play a major role in collateral growth (arteriogenesis) and angiogenesis. Monocyte involvement in inflammatory angiogenesis has already been reported (6). A role of monocytes/macrophages in the process of adaptive arteriogenesis was first suggested from our laboratory in studies where a correlation was shown between monocyte adhesion to the intima of coronary collaterals and rapid growth of these vessels (25). A few years later Polverini and Leibowitz demonstrated that peritoneal macrophages displayed angiogenic properties (6). Recently we were 

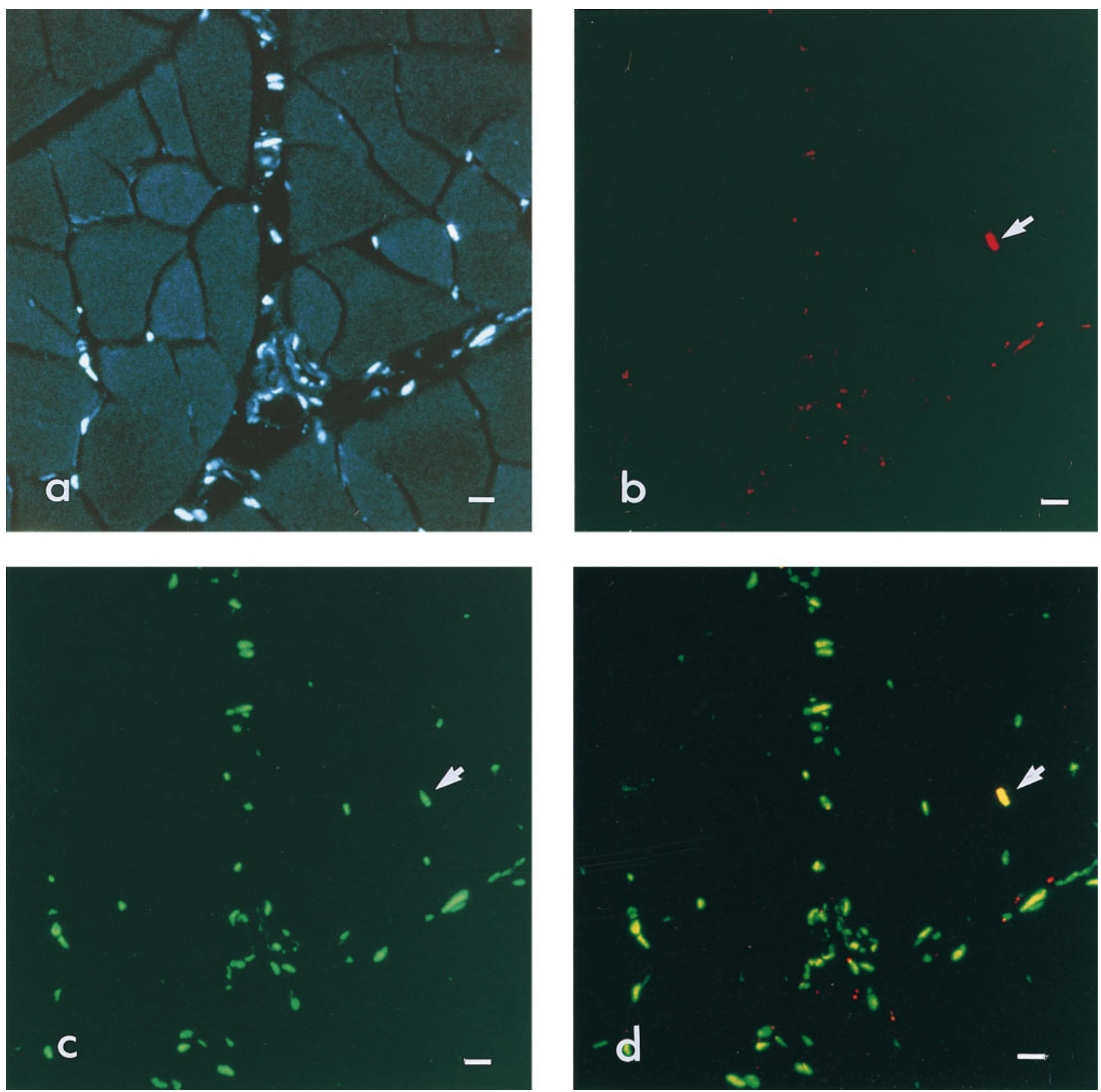

Figure 5. Double staining for bFGF and macrophages in the calf. (a) Morphology in the calf $7 \mathrm{~d}$ after ligation of the femoral artery. (b) Only a few macrophages occur in the lower leg after $7 \mathrm{~d}$ of ligation. (c) Specific staining for bFGF shows many positive cells in the interstitium. $(d)$ Double staining for bFGF and macrophages in the same section reveals a few positive cells for both antibodies (arrow), but numerous bFGF positive cells in the interstitium. Bars, $20 \mu \mathrm{m}$.

able to show that infusion of MCP-1 leads to a pronounced increase in collateral conductance and density of collateral vessels (5). Together with our present study, this result suggests that monocytes play an important role in peripheral arteriogenesis where they adhere to growing arterioles and provide angiogenic growth factors such as bFGF and TNF- $\alpha$. We furthermore show that LPS, the most potent stimulator of TNF- $\alpha$ production in monocytes and macrophages, enhances angiogenesis.

Monocytes attached selectively to and migrated through growing collateral arteries at the time of maximal proliferation after femoral artery occlusion. Only these monocytes/macrophages stained positively for bFGF as demonstrated by two different antibodies against bFGF. Rarely were other cells found to stain positively for bFGF. This result indicates that monocytes are the main source of bFGF, which has been shown to promote collateral vessel growth and angiogenesis $(13,26,27)$. bFGF is effective at very low concentrations that are not necessarily detectable immunohistochemically. Therefore, it is not surprising that we did not find any immunohistochemically detectable protein at or in endothelial or smooth muscle cells. 


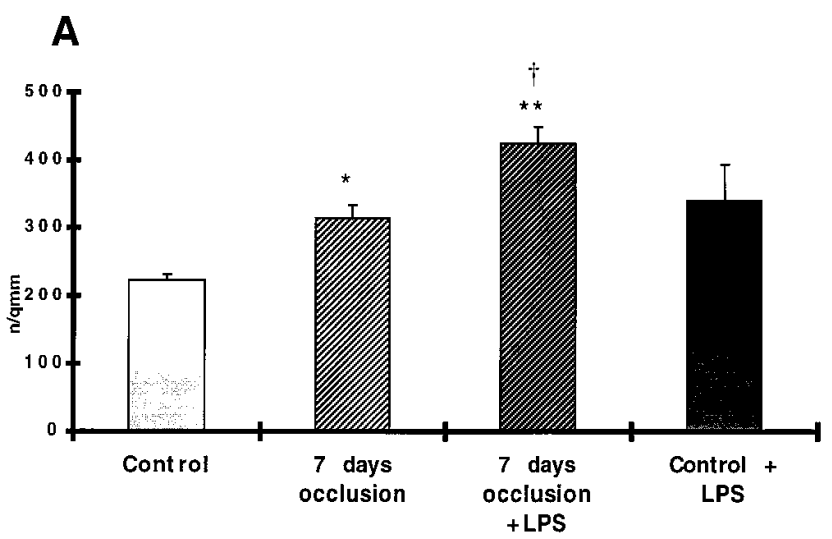

B

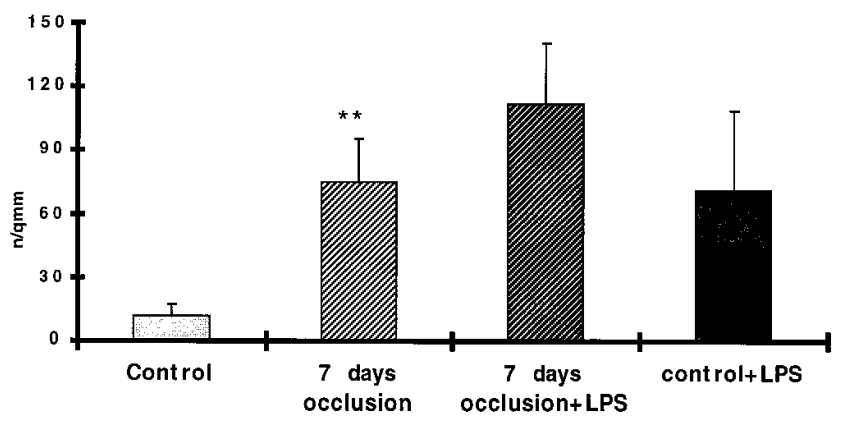

Figure 6. Number of capillaries and number of monocytes/macrophages in the gastrocnemial muscle of control animals without occlusion, after $7 \mathrm{~d}$ of occlusion, after $7 \mathrm{~d}$ of occlusion and LPS-treatment, and after LPS treatment without occlusion. $(A)$ Number of capillaries; $(B)$ number of monocytes/macrophages. ${ }^{*} * P<0.01$ and $* P<$ 0.05 versus control; ${ }^{\dagger} P<0.05$ versus $7 \mathrm{~d}$ of occlusion ( $n /$ qmm; number per square millimeter).

The adhering and migrating monocytes also stained positively for TNF- $\alpha$. This angiogenic cytokine is known to increase the expression of cell adhesion molecules $(18,28,29)$ and GM-CSF, and is thus principally capable of augmenting the monocytic response. Enhancement of vascular permeability as well as tissue factor production by $\mathrm{TNF}_{-\alpha}$ (30) may also contribute to the angiogenic properties of this cytokine. Increased vessel leakage and extravasation of plasma fibrinogen may aid in the formation of a new extracellular matrix and contribute significantly to collateral growth in a similar manner as that proposed for VEGF by Dvorak (31). Our findings therefore render an explanation for the rapid onset of collateral proliferation as well as for the early intensity of vascular growth.

The stimulus responsible for activation of the endothelium and the molecules necessary for selective adhesion of monocytes to growing collateral arteries as seen in our study are still unknown. In a previous study we were able to show that collateral growth occurred independently from perfusion deficiencies in the surrounding tissue (3). Therefore, a local stimulus different from hypoxia is needed to explain collateral growth in the thigh. It is likely that shear stress is this stimulus. Shear stress has been shown to upregulate production of cell adhe- sion molecules and monocyte chemoattractants like MCP-1 in vitro (32-34). Whether further cytokines such as VEGF and specific cell adhesion molecules are involved in collateral growth is the subject of ongoing research. In light of our findings suggesting that monocytes are critical players in angiogenesis as well as in collateral growth, the chemoattractant effect of VEGF needs further attention.

Capillary proliferation in the calf muscles was also associated with marked monocyte accumulation. In regions where no increase in monocytes/macrophages were noted after femoral artery occlusion (peroneal muscle), we also did not observe angiogenesis. Only an increase in the number of macrophages as seen in the gastrocnemial muscle seems to be associated with angiogenesis. Resident tissue macrophages that are also detectable by the antibody used in this study do not seem to be engaged in capillary sprouting, as fewer of these macrophages stained positive for bFGF.

In the calf region, macrophages were not the only sources of bFGF that were also produced by fibroblasts and myocytes. This result indicates that, in contrast to collateral growth, angiogenesis is elicited by factors that not only stimulate monocytes, but also stimulate other cells to produce growth factors. In previous observations, we found that the lower limb suffered from perfusion deficits, unlike the thigh, where collateral

\section{A}
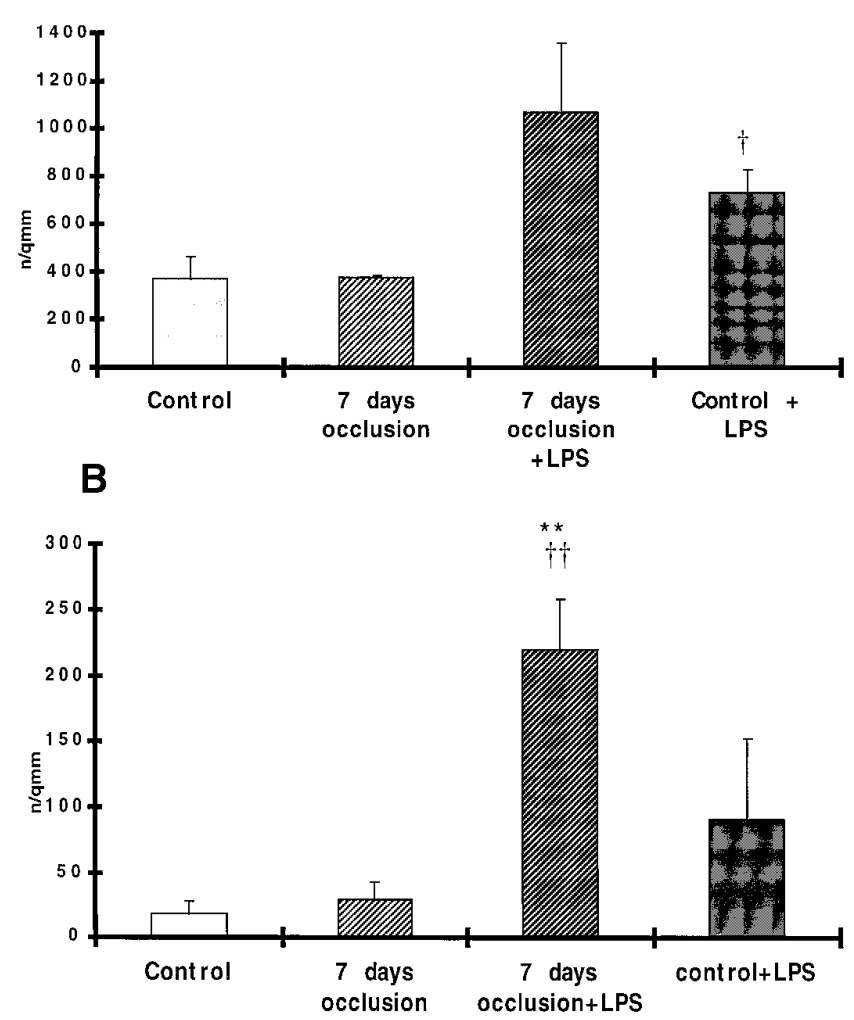

Figure 7. Number of capillaries and number of monocytes/macrophages in the peroneal muscle of control animals without occlusion, after $7 \mathrm{~d}$ of occlusion, after $7 \mathrm{~d}$ of occlusion and LPS treatment, and after LPS treatment without occlusion. $(A)$ Number of capillaries; $(B)$ number of monocytes/macrophages. $* * P<0.01$ versus control; ${ }^{\dagger} P<0.01$, and ${ }^{\dagger} P<0.05$ versus $7 \mathrm{~d}$ of occlusion.(n/qmm; number per square millimeter). 

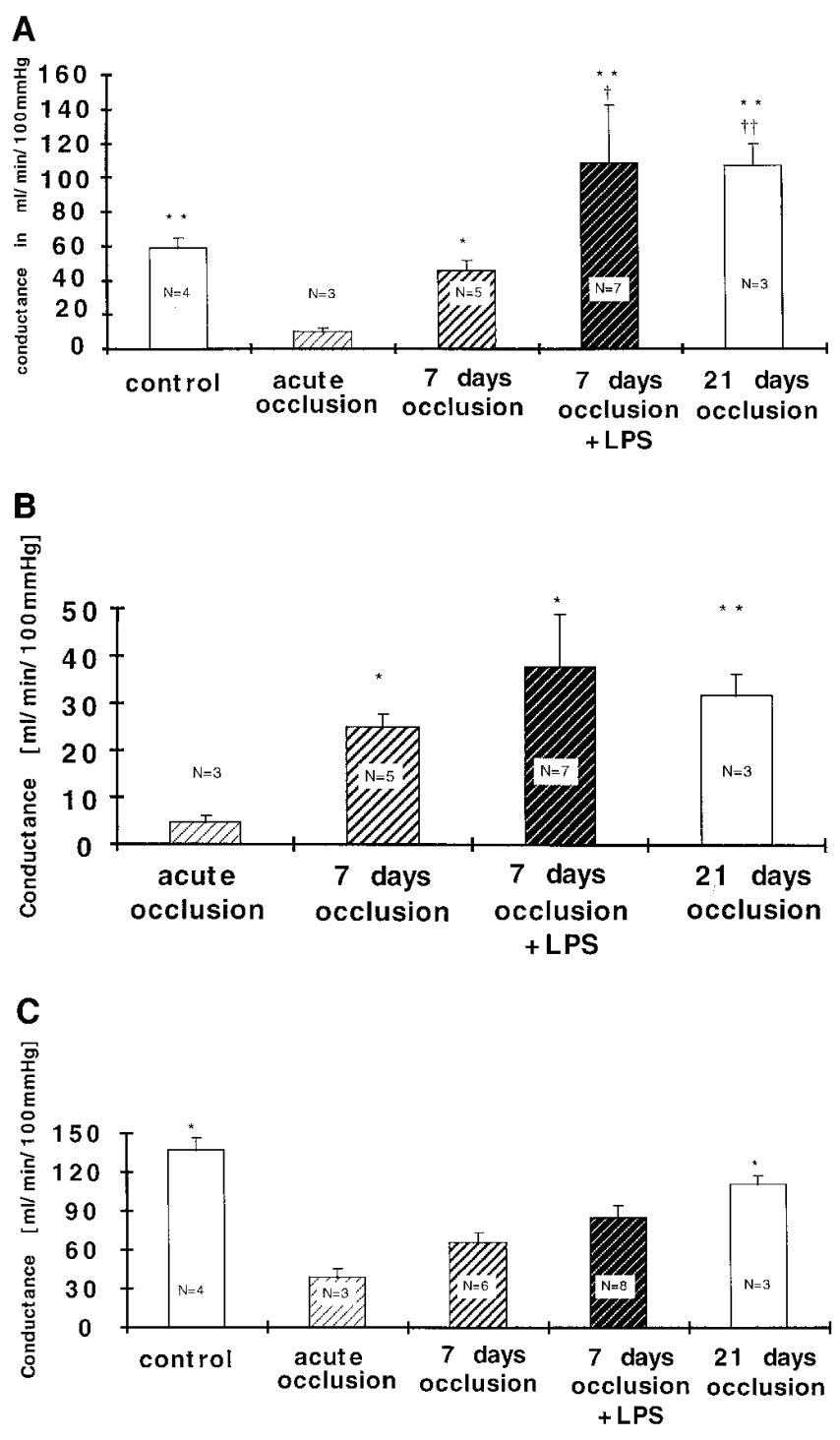

Figure 8. Conductances as calculated from pressure flow relations. $(A)$ Peripheral conductances; $(B)$ collateral conductances; $(C)$ bulk conductances. ${ }^{*} P<0.05$ and $* * P<0.01$ as compared with acute occlusion; ${ }^{\dagger} P<0.05$ and ${ }^{\dagger \dagger} P<0.01$ as compared with 1 wk of occlusion without LPS treatment.

growth and no perfusion deficits were found (3). Therefore, it is likely that hypoxia is the stimulus for angiogenesis leading to growth factor production in the hypoxic tissue. Some myocytes may not survive the hypoxic threat, therefore becoming necrotic. Monocytes that are activated to remove necrotic tissue may also contribute to angiogenesis. Indeed, induction of chemoattractant proteins leading to monocyte migration has recently been described for the ischemic myocardium (35). As in collateral arteries, macrophages in regions of capillary sprouting stained positively for $\mathrm{TNF}-\alpha$.

The role of TNF- $\alpha$ in angiogenesis and collateral growth was further studied by injecting a single dose of LPS, since this endotoxin is the most potent stimulus for TNF- $\alpha$ production in monocytes/macrophages $(20,21)$. This injection led to an increase in monocyte accumulation in the gastrocnemial and peroneal muscles, and concomitantly to an increase in the num- ber of capillaries. Further evidence that monocyte stimulation by LPS promotes angiogenesis comes from our hemodynamic studies that were performed ex vivo because we sought to investigate early events in collateral growth and angiogenesis; in vivo measurements as performed by others were not sensitive enough (36). We observed a significant increase in peripheral conductance, which we have shown to be a functional parameter of angiogenesis (3). Interestingly, LPS also leads to an increase in capillary density and an increase in the number of macrophages in nonoccluded hindlimbs. This effect was more pronounced in muscles with a higher density of resident tissue macrophages. Therefore, activation of resident macrophages and further accumulation of macrophages via TNF- $\alpha$ is a likely explanation for this phenomenon, and underscores the role monocytes play in capillary sprouting. Activation of monocytes by LPS at day 3 also led to an increase in collateral conductance that failed to reach statistical significance. This finding coincides with our observation that monocyte accumulation and proliferation had already reached their maximum at day 3 , and probably could not be stimulated much further. It may be speculated that at that time, continuous $\mathrm{TNF}_{-} \alpha$ production had already led to downregulation of its own receptors, and thus impeded the effect of LPS. Downregulation of TNF- $\alpha$ receptors by TNF- $\alpha$ and by LPS has been demonstrated before (37). As already mentioned above, timely administration of a specific chemoattractant for monocytes significantly increases collateral conductance and collateral vessel density (5).

In conclusion, monocytes accumulate during collateral artery growth and angiogenesis. They are the main source of TNF- $\alpha$ and bFGF in proliferating collateral arteries. Enhancement of monocyte accumulation by LPS promotes angiogenesis and collateral growth. These results add further evidence to the concept that monocyte accumulation and activation play a major role in angiogenesis and collateral arteriogenesis.

\section{Acknowledgments}

Wulf D. Ito was supported by a postdoctoral research scholarship from the German Cardiac Society.

\section{References}

1. Risau, W. 1997. Mechanisms of angiogenesis. Nature. 386:671-674.

2. Schaper, W., and W.D. Ito. 1996. Molecular mechanisms of coronary collateral vessel growth. Circ. Res. 79:911-919.

3. Ito, W.D., M. Arras, D. Scholz, B. Winkler, P. Htun, and W. Schaper. 1997. Angiogenesis but not collateral growth is associated with ischemia after femoral artery occlusion. Am. J. Physiol. 273:H1255-H1265.

4. White, F.C., S.M. Carroll, A. Magnet, and C.M. Bloor. 1992. Coronary collateral development in swine after coronary artery occlusion. Circ. Res. 71: 1490-1500.

5. Ito, W.D., M. Arras, B. Winkler, D. Scholz, J. Schaper, and W. Schaper. 1997. Monocyte chemotactic protein-1 increases collateral and peripheral conductance after femoral artery occlusion. Circ. Res. 80:829-837.

6. Polverini, P.J., R.S. Cotran, M.A. Gimbrone, and E.R. Unanue. 1977. Activated macrophages induce vascular proliferation. Nature. 269:804-806.

7. Berse, B., L.F. Brown, L.V.D. Water, H.F. Dvorak, F. Dvorak, and D.R. Senger. 1992. Vascular permeability factor (vascular endothelial growth factor) gene is expressed differentially in normal tissues, macrophages and tumors. Mol. Biol. Cell. 3:211-220.

8. Wahl, S.M., D.A. Hunt, L.M. Wakefield, N. McCartney-Francis, L.M. Wahl, A.B. Roberts, and M.B. Sporn. 1987. Transforming growth factor type $\beta$ induces monocyte chemotaxis and growth factor production. Proc. Natl. Acad. Sci. USA. 84:5788-5792.

9. Iijima, Z., N. Yoshikawa, D.T. Connolly, and H. Nakamura. 1993. Human mesangial cells and peripheral blood mononuclear cells produce vascular permeability factor. Kidney Int. 44:959-966.

10. Fràter-Schröder, M., W. Risau, R. Hallmann, P. Gautschi, and P. 
Böhlen. 1987. Tumor necrosis factor type alpha, a potent inhibitor of endothelial cell growth in vitro, is angiogenic in vivo. Proc. Natl. Acad. Sci. USA. 84: 5277-5281.

11. Leibovich, S.J., P.J. Polverini, H.M. Shepard, D.M. Wiseman, V. Shively, and N. Nuseir. 1987. Macrophage-induced angiogenesis is mediated by tumor necrosis factor-alpha. Nature. 329:630-632.

12. Wahl, S.M., H. Wong, and N. McCarney-Francis. 1989. Role of growth factors in inflammation and repair. J. Cell. Biochem. 40:193-199

13. Lazarous, D.F., M. Scheinowitz, M. Shou, E. Hodge, S. Rajanayagam, S. Hunsberger, W.G.J. Robinson, J.A. Stiber, R. Correa, and S.E. Epstein. 1995. Effects of chronic systemic administration of basic fibroblast growth factor on collateral development in the canine heart. Circulation. 91:145-153.

14. Goto, F., K. Goto, K. Weindel, and J. Folkman. 1993. Synergistic effects of vascular endothelial growth factor and basic fibroblast growth factor on the proliferation and cord formation of bovine capillary endothelial cells within collagen gels. Lab. Invest. 69:508-517.

15. Bastagli, L., T. Lazzarotto, C.M. Caldarera, C. Guarnieri, C. Ventura, G. Pepe, and P. Puddu. 1995. Presence of basic fibroblast growth factor in cultured rat cardiomyocytes and its release in culture medium. Ann. NY Acad. Sci. 752:417-421.

16. Riese, J., R. Zeller, and R. Dono. 1995. Nucleo-cytoplasmic translocation and secretion of fibroblast growth factor-2 during avian gastrulation. Mech. Dev. 49:13-22.

17. Lupia, E., G. Montrucchio, E. Battaglia, V. Modena, and G. Camussi. 1996. Role of tumor necrosis factor-alpha and platelet-activating factor in neoangiogenesis induced by synovial fluids of patients with rheumatoid arthritis. Eur. J. Immunol. 26:1690-1694.

18. Gamble, R.G., W.B. Smith, and M.A. Vadas. 1992.TNF modulation of endothelial and neutrophil adhesion. In Tumor Necrosis Factors: The Molecules and Their Emerging Role in Medicine. B. Beutler, editor. Raven Press, Ltd., New York. 65-86.

19. Ross, H.J., and H.P. Koeffler. 1992. Interactions between TNF and GMCSF. In Tumor Necrosis Factors: The Molecules and Their Emerging Role in Medicine. B. Beutler, editor. Raven Press, Ltd., New York. 179-196.

20. Vogel, S.N. 1992.The Lps gene: insights into the genetic and molecular basis of LPS responsiveness and macrophage differentiation. In Tumor Necrosis Factors: The Molecules and Their Emerging Role in Medicine. B. Beutler, editor. Raven Press, Ltd., New York. 485-513.

21. Arras, M., A. Höche, R. Bohle, P. Eckert, W. Riedel, and J. Schaper. 1996. Tumor necrosis factor-alpha in macrophages of heart, liver, kidney and in the pituitary gland. Cell Tissue Res. 285:39-49.

22. Winkler, B. 1984. Measurement of coronary blood flow. In Techniques in the Life Sciences. R.J. Linden, editor. Elsevier Science Ireland Ltd., Limerick, Ireland. P1-P36.

23. Fulton, W.F.M. 1965. The Coronary Arteries. Charles C. Thomas, Springfield, Illinois.

24. Cortese, R., P. Monaci, A. Luzzago, C. Santini, F. Bartoli, I. Cortese, P. Fortugno, G. Galfre, A. Nicosia, and F. Felici. 1996. Selection of biologically active peptides by phage display of random peptide libraries. Curr. Opin Biotechnol. 7:616-621.
25. Schaper, J., R. Koenig, D. Franz, and W. Schaper. 1976. The endothelial surface of growing coronary collateral arteries. Intimal margination and diapedesis of monocytes A combined SEM and TEM study. Virchows Arch. A Pathol. Anat. Histopathol. 370:193-205.

26. Unger, E.F., S. Banai, M. Shou, D.F. Lazarous, M.T. Jaklitsch, M. Sheinowitz, R. Correa, C. Klingbeil, and S.E. Epstein. 1994. Basic fibroblast growth factor enhances myocardial collateral flow in a canine model. Am. J. Physiol. 266:H1588-H1595.

27. Yanagisawa-Miwa, K., Y. Ushida, F. Nakamura, T. Tomaru, H. Kido, T. Kamijo, T. Sugimoto, K. Kaji, M. Utsuyama, C. Kurashima, and H. Ito. 1992. Salvage of infarcted myocardium by angiogenic action of basic fibroblast growth factor. Science 257:1401-1403.

28. Neumann, B., T. Machleidt, A. Lifka, K. Pfeffer, D. Vestweber, T.W. Mak, B. Holzmann, and M. Krönke. 1996. Crucial role of 55-kilodalton TNF receptor in TNF-induced adhesion molecule expression and leukocyte organ infiltration. J. Immunol . 156:1587-1593.

29. Mackay, F., H. Loetscher, D. Strueber, G. Gehr, and W. Lesslauer. 1993. Tumor necrosis factor alpha (TNF-alpha)-induced cell adhesion to human endothelial cells is under dominant control of one TNF receptor type TNF-R55. J. Exp. Med. 177:1277-1286.

30. Clauss, M., M. Grell, C. Fangman, W. Fiers, P. Scheurich, and W. Risau. 1996. Synergistic induction of endothelial tissue factor by tumor necrosis factor and vascular endothelial growth factor: functional analysis of the tumor necrosis factor receptors. FEBS Lett. 390:334-338.

31. Dvorak, H.F., L.F. Brown, M. Detmar, and A.M. Dvorak. 1995. Vascular permeability factor/vascular endothelial growth factor, microvascular hyperpermeability and angiogenesis. Am. J. Pathol. 146:1029-1039.

32. Patrick, C.W., and L.V. McIntire. 1995. Shear stress and cyclic strain modulation of gene expression in vascular endothelial cells. Blood Purif. 13: 112-124.

33. Shyy, Y.J., H.J. Hsieh, S. Usami, and S. Chien. 1994. Fluid shear stress induces a biphasic response of human monocyte chemotactic protein-1 gene expression in vascular endothelium. Proc. Natl. Acad. Sci. USA. 91:4678-4682.

34. Wung, B.S., J.J. Cheng, Y.F. Chao, J. Lin, Y.J. Shyy, and D.L. Wang. 1996. Cyclical strain increases monocyte chemotactic protein-1 secretion in human endothelial cells. Am. J. Physiol. 270:H1462-H1468.

35. Birdsall, H.H., D.M. Green, J. Trial, K.A. Youker, A.R. Burns, C.R Mackay, G.J. LaRosa, H.K. Hawkins, C.W. Smith, L.H. Michael, et al. 1997. Complement D5a, TGF- $\beta 1$ and MCP-1, in seyuence, induce migration of monocytes into ischemic canine myocardium within the first one to five hours after reperfusion. Circulation. 95:684-692.

36. Takeshita, S., L.P. Zheng, E. Brogi, M. Kearney, L.-Q. Pu, S. Bunting, N. Ferrara, J.F. Symes, and J.M. Isner. 1994. Therapeutic angiogenesis. A single intraarterial bolus of vascular endothelial growth factor augments revascularization in a rabbit ischemic hind limb model. J. Clin. Invest. 93:662-670.

37. van der Poll, T., S.M. Coyle, A. Kumar, K. Barbosa, J.M. Agosti, and S.F. Lowry, 1997. Down-regulation of surface receptors for TNF and IL-1 on circulating monocytes and granulocytes during human endotoxemia. J. Iтmиnol. 158:1490-1497. 